\title{
GaN quantum dot polarity determination by X-ray photoelectron diffraction
}

\author{
O. Romanyuk ${ }^{a, *}$, I. Bartoš ${ }^{a}$, J. Brault ${ }^{b}$, P. De Mierry ${ }^{b}$, T. Paskova ${ }^{c}$, P. Jiříček ${ }^{a}$ \\ ${ }^{a}$ Institute of Physics, Academy of Sciences of the Czech Republic, Cukrovarnická 10, \\ CZ-162 00 Prague 6, Czech Republic \\ ${ }^{b}$ CRHEA-CNRS, Centre de Recherche sur l'Hétéro-Epitaxie et ses Applications, Centre \\ National de la Recherche Scientifique, Rue B. Grégory, Valbonne 06560, France \\ ${ }^{c}$ Department of Electrical and Computer Engineering, North Carolina State University, \\ Raleigh, North Carolina 27606, USA
}

\begin{abstract}
Growth of GaN quantum dots (QDs) on polar and semipolar GaN substrates is a promising technology for efficient nitride-based light emitting diodes (LED) due to suppressed dislocation density in the active region of the devices. The QDs crystal orientation typically repeats the polarity of the substrate. In case of non-polar or semipolar substrates, the polarity of QDs is not obvious. In this article, the polarity of GaN QDs and of underlying layers was investigated nondestructively by X-ray photoelectron diffraction (XPD). Polar and semipolar $\mathrm{GaN} / \mathrm{Al}_{0.5} \mathrm{Ga}_{0.5} \mathrm{~N}$ heterostructures were grown on the sapphire substrates with (0001) and (1100) orientations by molecular beam epitaxy (MBE). Polar angle dependence of $\mathrm{N}$ 1s core-level photoelectron intensities were measured from GaN QDs and compared with the corresponding experimental curves from freestanding GaN crystals. It is confirmed experimentally, that the crystalline orientation of polar (0001) GaN QDs follows the orientation of the (0001) sapphire substrate. In case of semipolar GaN QDs grown on (1100) sapphire substrate, the $(11 \overline{2} 2)$ polarity of QDs was determined.
\end{abstract}

Keywords: GaN, semipolar GaN, quantum dots, X-ray photoelectron diffraction, surface polarity

\footnotetext{
${ }^{*}$ Corresponding author

Email address: romanyuk@fzu.cz (O. Romanyuk)
}

Preprint submitted to Elsevier

July 19, 2016

(C) 2016. This manuscript version is made available under the Elsevier user license http://www.elsevier.com/open-access/userlicense/1.0/ 


\section{Introduction}

Nitride semiconductors are nowadays used for the fabrication of a wide range of optoelectronic devices such as white light emitting diodes (LEDs) for lighting applications or blue laser diodes. More recently, aluminium gallium nitride $\left(\mathrm{Al}_{x} \mathrm{Ga}_{1-x} \mathrm{~N}\right)$ based LEDs emitting in the ultra-violet (UV) range have attracted a great interest aiming a replacement of the mercury lamp, which faces the severe toxicity issue in addition to technological issues. In particular, the possibility to tune the wavelength from 200 to $360 \mathrm{~nm}$ by simply adjusting the $\mathrm{Al}$ concentration of $\mathrm{Al}_{x} \mathrm{Ga}_{1-x} \mathrm{~N}$ materials makes them perfectly suitable for this goal.

Although the performance of the UV LEDs has been recently significantly improved [1], their external quantum efficiency (EQE) is still noticeably lower than that of blue LEDs. This observation was attributed to the structural quality of $\mathrm{Al}_{x} \mathrm{Ga}_{1-x} \mathrm{~N}$ materials, mainly grown on sapphire substrates, with typical dislocation densities (DDs) in the range of $10^{8}-10^{10} \mathrm{~cm}^{-2}$, leading to low radiative efficiencies. Different approaches employing various $\mathrm{Al}_{x} \mathrm{Ga}_{1-x} \mathrm{~N}$ growth process optimizations [2] or bulk AlN substrates [3] have been developed, leading to a significant improvement of the EQE value of UV LEDs. Noteworthy, this is also the typical DD range for highly efficient blue InGaN LEDs; one explanation is that (In,Ga)N quantum wells (QWs) are less affected by dislocations than UV $(\mathrm{Al}, \mathrm{Ga}) \mathrm{N}$ QWs, due to local indium fluctuations.

Along this view, another attractive solution to overcome the negative impact of the high DDs is to spatially confine carriers in the three spatial directions, i.e. through the use of quantum dots (QDs) instead of the classical unidirectional confinement in QWs [4]. Indeed, $\mathrm{Al}_{y} \mathrm{Ga}_{1-y} \mathrm{~N}$ QDs can be grown on $\mathrm{Al}_{x} \mathrm{Ga}_{1-x} \mathrm{~N}$ layers by triggering a three dimensional (3D) growth mode during the epitaxial process [5, 6, 7]. Taking advantage of the compressive epitaxial stress between $\mathrm{Al}_{y} \mathrm{Ga}_{1-y} \mathrm{~N}$ and $\mathrm{Al}_{x} \mathrm{Ga}_{1-x} \mathrm{~N}$ (with $\mathrm{x}>\mathrm{y}$ ), $\mathrm{Al}_{y} \mathrm{Ga}_{1-y} \mathrm{~N}$ QDs are formed through a 2D - 3D growth mode transition by molecular beam epitaxy (MBE). Using this 
approach, QD-LEDs emitting in the near UV range have been demonstrated [8], with ability to tune the LED wavelength by modifying the QDs structural properties and to assemble QDs into objects with varying dimensionality [9, 10].

Importantly, GaN crystals have a wurtzite structure, which consists of two shifted $\mathrm{Ga}$ and $\mathrm{N}$ hexagonal close-packed sublattices with AB bilayer stacking sequence along the [0001] direction. The crystal lacks the inversion symmetry and two crystal terminations are possible, i.e. either cation atoms on top of a Ga$\mathrm{N}$ bilayer along [0001] direction ( $+\mathrm{c}$ polarity) or anion atoms on top of a N-Ga bilayer along the $[000 \overline{1}]$ direction (-c polarity). The polarity of the epitaxial GaN films or nanostructures is an important characteristic, influencing the growth conditions [11], the structural properties [12] as well as the optical properties of the devices [13]. Both piezoelectric and spontaneous polarization fields along the [0001] direction in GaN crystal can reduce the electron-hole wave function overlap in quantum well devices and decrease the radiative recombination rate and the light efficiency in the devices. The direction of the internal fields in GaN depends on the structure polarity, and, therefore, it is technologically important to control the nanostructure growth-front polarity. Along these views, the QDs fabrication process makes it possible to grow QDs on both (0001) oriented 'polar' and $(11 \overline{2} 2)$ oriented 'semipolar' $\mathrm{Al}_{x} \mathrm{Ga}_{1-x} \mathrm{~N}$ surfaces. In particular, by using semipolar orientations, the internal electric field can be drastically reduced as compared to the polar (0001) surface, which enables the emission shift to shorter wavelengths [14]. Using this approach we were able to fabricate (11̄22) QD-based UV LEDs emitting at much shorter wavelengths than their (0001) oriented counterparts [15].

It should be emphasized that the conventional X-ray diffraction (XRD) does not provide information about wurtzite GaN crystal polarity. Alternatively, there are a variety of alternative methods for the identification of wurtzite crystal polarity. The easiest but destructive method for polarity determination of polar GaN surfaces or GaN nanowires is etching in $\mathrm{KOH}$ solutions [16, 17]. Other known methods are convergent beam electron diffraction (CBED) 18, 19, 20, 12], electron energy loss spectroscopy [18, 21] piezoresponse force mi- 
croscopy [22], photoassisted Kelvin probe force microscopy [23], resonant XRD with tunable monochromatic synchrotron radiation [19], and scanning transmission electron microscopy (TEM) [24]. The high-resolution TEM in the Zcontrast imaging mode with a probe-Cs-corrector seems to be very useful for the wurtzite QDs lattice polarity determination directly without any image simulation, by contrast to what is necessary when using CBED technique [25]. All of these techniques, however, are either destructive, local or require complex sample preparation procedures.

Recently, we have developed a relatively simple non-destructive X-ray photoelectron diffraction (XPD) based technique for GaN polarity analysis. Polarity of polar [26] and semipolar [27, 28] free-standing GaN crystals was determined using Mg K $\alpha$ source (photon energy $1253 \mathrm{eV}$ ) of a typical X-ray photoelectron spectroscopy (XPS) tool. The method is based on the fact that the spatial distribution of neighboring atoms differs for the two opposite GaN crystal orientations and polar angle dependence of photoelectron intensities exhibits local enhancements in the directions toward their atomic neighbors, which is different for opposite crystal orientations [28]. For wurtzite GaN epitaxial films or free-standing crystal surfaces, the photoelectron emission from $\mathrm{N}$ atoms appeared most suitable for polarity determination. In addition to crystal surfaces, the approach has also been successfully applied for polarity determination of GaN nanowires grown by plasma-assisted MBE on polar and non-polar substrates [17].

In this article, polarity of polar and semipolar GaN QDs is analyzed nondestructively by XPD method. In contrast to our previous studies on clean surfaces, in this work two samples were measured 'as-received' without additional surface cleaning procedures. Due to limited inelastic mean free path (IMFP) of photoelectrons, only a few surface layers of QDs and substrate layers were investigated. Despite surface contamination, we were able to measure polar angle dependences of photoemission current in high symmetry azimuthal directions of the GaN QDs and, by using a comparative analysis with the corresponding dependencies of the free-standing GaN, to determine the GaN QDs polarity. 
(a) $\mathrm{S1}$

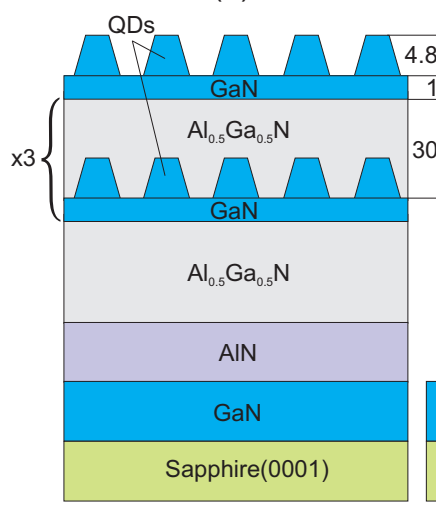

(b) S2

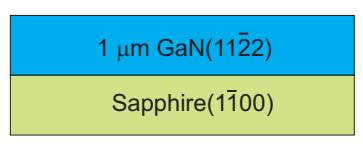

(c) S3

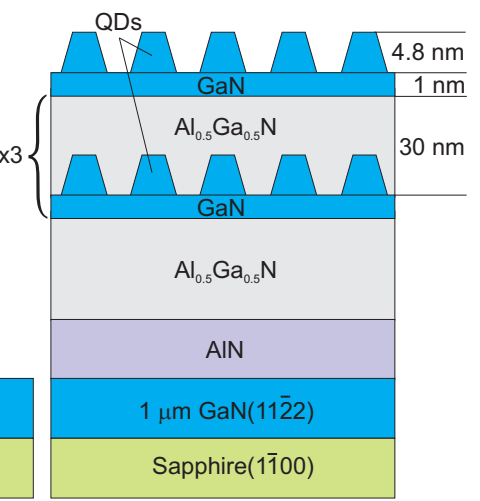

Figure 1: (Color online) Schematic view of the samples used for the analysis. Sample (a) S1 was grown on sapphire (0001) and terminated by the polar GaN QDs. Samples (b) S2 and (c) $\mathrm{S} 3$ were grown on sapphire (1100). The $\mathrm{S} 2$ is covered by the epitaxial $\mathrm{GaN}\{11 \overline{2} 2\}$ film only, whereas the S3 consists of heterotructures on top of S2 and terminated by the semipolar GaN QDs.

\section{Experimental details}

Three types of samples were grown for this analysis. In Fig. 1 the following heterostructures are schematically shown: (a) S1 sample is terminated by $\mathrm{GaN}\{0001\}$ QDs on top of $\mathrm{GaN} / \mathrm{Al}_{0.5} \mathrm{Ga}_{0.5} \mathrm{~N}$ layers deposited on sapphire (0001) substrate; (b) S2 is a reference sample with epitaxial semipolar GaN $\{11 \overline{2} 2\}$ film grown on sapphire (11̄00); (c) S3 sample contains the semipolar $\operatorname{GaN}\{11 \overline{2} 2\}$ QDs on top of semipolar $\mathrm{GaN} / \mathrm{Al}_{0.5} \mathrm{Ga}_{0.5} \mathrm{~N}$ layers grown on sapphire (11̄00) .

The polar (S1) and semipolar (S3) QD samples grown on (0001) c-plane and (1100) m-plane sapphire substrates, respectively, include the following growth specifics: At first, a GaN buffer layer was grown. MBE was used for the growth on c-plane sapphire and metal organic vapor phase epitaxy (MOVPE) was used for the growth on m-plane sapphire. Then, the following layers were grown on S1 and S3 samples by MBE (RIBER 32) reactor using ammonia $\left(\mathrm{NH}_{3}\right)$ as nitrogen precursor, and solid sources for the III-elements: an AlN layer grown on top of the GaN buffer layer, followed by a $0.8-\mu$ m-thick $\mathrm{Al}_{0.5} \mathrm{Ga}_{0.5} \mathrm{~N}[5$, 14]. Then, three GaN QD planes separated by $30-\mathrm{nm}$-thick $\mathrm{Al}_{0.5} \mathrm{Ga}_{0.5} \mathrm{~N}$ barrier layers and a 

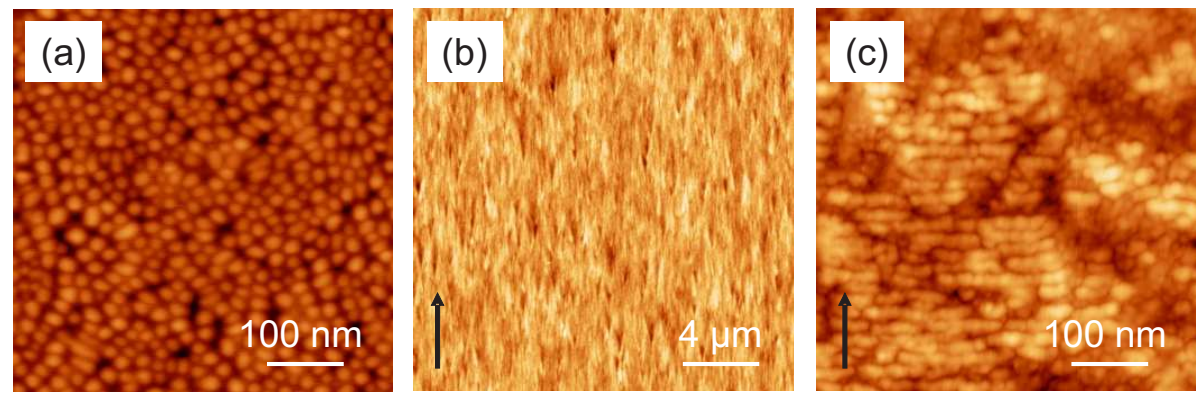

Figure 2: (Color online) AFM images of (a) S1, (b) S2, and (c) S3 samples. QDs cover around $30 \%$ of the surface area for sample S1 and around $50 \%$ of sample S3, respectively. A root mean square roughness (RMS) value of $5.6 \mathrm{~nm}$ was determined for the $\mathrm{S} 2$ sample. The vertical scale for the images is zero for dark and $10 \mathrm{~nm}, 60 \mathrm{~nm}$ and $10 \mathrm{~nm}$ for light, respectively. The black arrow in figs. (a) and (c) indicates the $<\overline{1} \overline{1} 23>$ direction.

last uncapped QD layer on the top were grown. Atomic force microscopy (AFM) measurements were performed to study the uncapped QD layer morphology.

Representative AFM images of the three samples are shown in Fig. 2. The morphology of sample S1 [Fig. 2(a)] reveals uniform QD distribution with high QD density of $\sim 1.1 \times 10^{11} \mathrm{~cm}^{-2}$. The QDs on the polar GaN possesses a hexagonal base [29], with an average height of $4.8 \mathrm{~nm}$ and an average diameter of 30 $\mathrm{nm}$. The QD surface coverage area for sample $\mathrm{S} 1$ is around 0.3 . The morphology of the 1- $\mu$ m-thick (112̄2) GaN film [sample S2, Fig. 2(b)] reveals elongated domains aligned along the $<\overline{1} \overline{1} 23>$ axis with a clear anisotropy between the $<1 \overline{1} 00>$ and the $<\overline{1} \overline{1} 23>$ in-plane directions. In addition, stripes and undulations are observed along the $<\overline{1} \overline{1} 23>$ direction with a period of $\sim 200 \mathrm{~nm}$ along the $<1 \overline{1} 00>$ direction with a shorter period of $\sim 100 \mathrm{~nm}[9]$. The S2 sample was covered by GaN completely. Finally, the morphology of sample S3 [Fig. 2 (c)] reveals similar high density of QDs $\sim 2 \times 10^{11} \mathrm{~cm}^{-2}$. The QDs grown on the semipolar layers possesses an elongated shape along the $<1 \overline{1} 00>$ direction with an average height of $3 \mathrm{~nm}$, and average width of $20 \mathrm{~nm}$. They are also aligned along this axis, forming strings of QDs [14] with an average length of $40 \mathrm{~nm}$. In the sample S3, the QD surface coverage area is around 0.5. 
The GaN surfaces were cleaned ex-situ in isopropyl alcohol prior to loading into an ADES-400 angular resolved photoelectron spectrometer. To check a cleanliness of the samples, XPS measurements were performed with base pressure in the spectrometer chamber of $2 \times 10^{-9}$ mbar. Detected oxygen concentration was less than 25 at\% on S1 and S2 sample surfaces. The oxygen concentration on S3 sample surface was 37 at\%. Carbon contamination was not analyzed because C 1s photoelectron peak overlaps with Ga LMM Auger line for $\mathrm{Mg}$ K $\alpha$ X-ray source. The S1 and S2 samples were measured 'as-received', while the $\mathrm{S} 3$ sample was annealed at $590^{\circ} \mathrm{C}$ in $\mathrm{NH}_{3}$ atmosphere $\left(1.5 \times 10^{-6}\right.$ mbar $)$ during 240 minutes in order to decrease the surface contaminations. After annealing, oxygen concentration decreased to 22 at\% on the S3 sample surface, enabling appearance of weak low-energy electron (LEED) patterns.

The LEED patterns were used for determining the QD sample alignment. In Fig. 3. LEED patterns measured on (a) S1, (b) S2, (c) S3 samples and (d) freestanding (FS) GaN(0001) crystal [28] are shown. Despite the high background intensity due to surface contamination, a six-fold symmetry LEED pattern is clearly seen for as-received S1 sample [Fig. 3 (a)]. This pattern corresponds to the $\{0001\}$ orientation of GaN crystal consistent with previous reports [26, 17]. The m-plane or $[11 \overline{2} 0]$ azimuthal direction lie between the hexagonally-aligned reflection maxima [30] and is marked by a dashed line in Fig. 3 (a). The XPD polar plots were measured along the $[11 \overline{2} 0]$ azimuthal direction.

The semipolar LEED patterns are more complex due to expected facets on semipolar GaN surfaces [31, 32]. Even for a FS-GaN $\{11 \overline{2} 2\}$ clean surface, a $(1 \times 1)$ unit cell is not well resolved. Nevertheless, there is an obvious similarity of S2 and S3 LEED pattern symmetry with the corresponding LEED pattern symmetry of the FS-GaN $(11 \overline{2} 2)$ sample [Fig. 3 (b, c, d)]. In combination with XPD measurements [28], azimuthal directions on $\{11 \overline{2} 2\}$ LEED patterns were assigned and the QD samples were aligned for further XPD measurements along the $[1 \overline{1} 00]$ and $[\overline{1} \overline{1} 23]$ directions.

The XPD measurements of the Ga 3d, Al 2p and N 1s core levels were carried out. The photon beam spot has a circular shape with diameter of around 10 
(a) S1

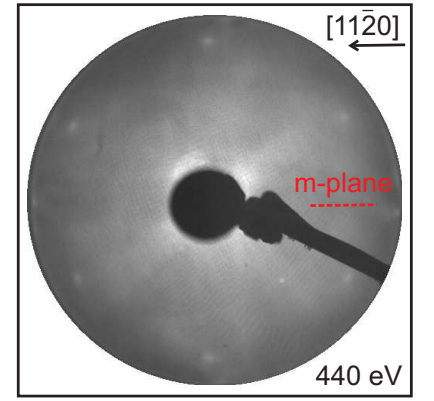

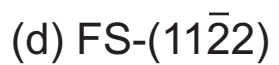

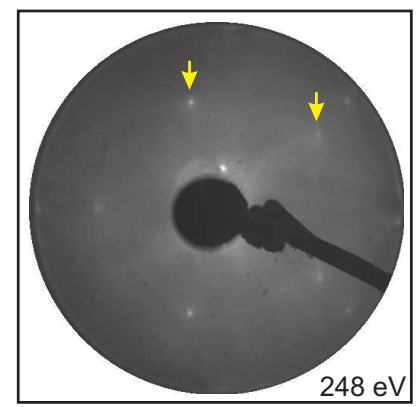

(b) S2

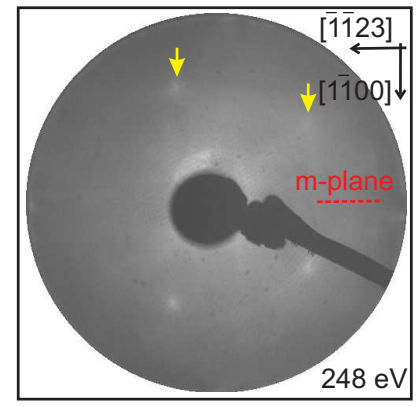

(c) $\mathrm{S3}$

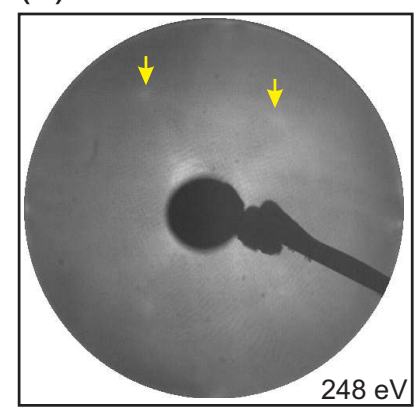

Figure 3: (Color online) LEED patterns from sample (a) S1 with primary electron beam energy of $440 \mathrm{eV}$; (b) S2, (c) S3, and (d) free-standing GaN(11̄̄2) with energy of $248 \mathrm{eV}$. Hexagonal pattern symmetry confirms the $\{0001\}$ orientation in (a). The m-plane lies between the reflections along the [11̄̄0] direction [30]. LEED patterns of S2 and S3 samples are more diffuse than the corresponding patterns of FS-(112̄2) sample. Spot positions in (d) (yellow arrows) was used for S2 and S3 samples alignment. XPD polar plots were measured along the

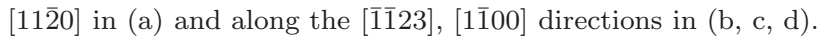

mm. The photoelectrons were collected from the almost entire sample surface over acceptance angle of the energy analyzer $\pm 2.1^{\circ}$. The photoelectron spectra were recorded with electron energy analyzer pass energy of $100 \mathrm{eV}$ and overall energy resolution of $1.72 \mathrm{eV}$. The incident X-ray beam impinged on the surface at $70^{\circ}$ with respect to the surface normal and photoemission angle (analyzer angle) was varied from the surface normal to $50^{\circ}$ with a step of $2^{\circ}$.

Polar angle dependences of the photoelectron intensities were obtained from the areas under each photoelectron peak. Shirley background was subtracted 
from the peaks and the peak integral intensity was computed by peak fitting by Gaussian functions. The integrated photoelectron intensities were normalized to the normal emission intensity.

\section{Results and Discussions}

In sample $\mathrm{S} 1$, a thin layer of GaN (thickness around $1 \mathrm{~nm}$ ) separates the QDs from the underlying thicker layer of $\mathrm{Al}_{0.5} \mathrm{Ga}_{0.5} \mathrm{~N}$ [Fig. 1 (a)]. Since the photoelectron intensity is attenuated during the electron propagation to the sample surface [33], the escape depth of the photoemitted electrons is typically about three times larger than the IMFP. For N 1s photoelectrons, the IMFP is about $1.5 \mathrm{~nm}$, and the photoemission signal is expected to originate from a depth of about $5 \mathrm{~nm}$.

The polarity of GaN $\{0001\}$ crystalline layers was determined from the polar plots of $\mathrm{N}$ 1s intensities within the m-plane, i.e. (1100) plane, of the crystal. According to the XPD measurements on the free-standing GaN crystals, a photoelectron intensity increase or decrease is expected in the range of $20^{\circ}-25^{\circ}$ for $(000 \overline{1})$ or (0001) polarity, respectively [17]. In order to determine the polarity of the QDs, the experimental XPD polar plots of N 1s photoelectron intensities are compared with the corresponding plots obtained from free-standing GaN(0001) and $(000 \overline{1})$ crystals [Fig. 4(a)]. For the [0001] polarity, a maximum at around

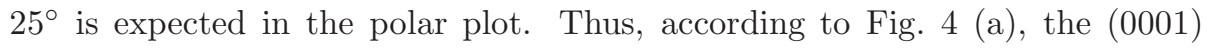
polarity of the QDs of S1 sample is confirmed.

Considering the fact that the QDs surface coverages were 30\% (sample $\mathrm{S} 1$ ) and $50 \%$ (sample S3), it is clear that the collected photoelectron emission comes from both the QDs and the GaN layers below the QDs. We were not able to separate these two contributions due to the large photon beam size of the standard XPS source. Nevertheless, in case of mixed polarity of QDs and base GaN layers, the peak between $20^{\circ}-25^{\circ}$ of S1 curve should shift towards high polar angles. Since this is not the case and since the curve from S1 sample agrees very well with the polar plot of the FS-GaN(0001) sample, the dominant 
(a) N1s, $[11 \overline{2} 0]$

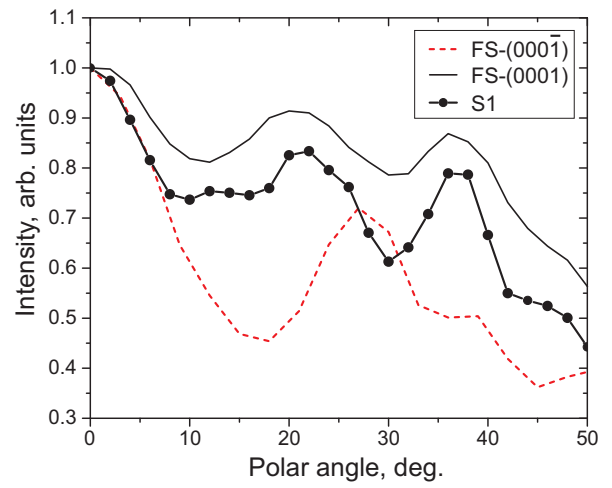

(b) Ga3d, Al2p, [1120]

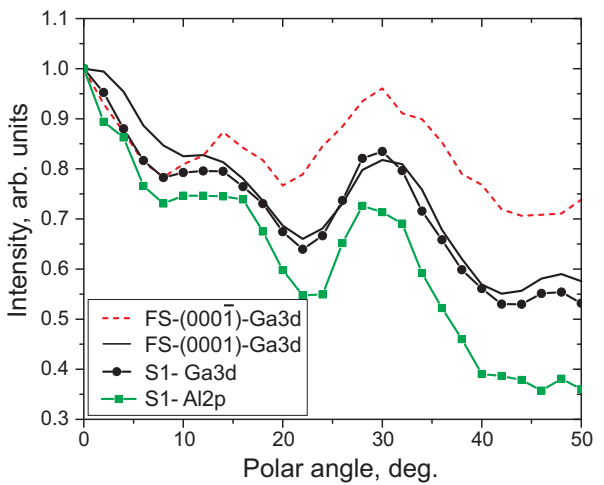

Figure 4: (Color online) (a) Experimental polar plots of N 1s XPD intensity measured along the $[11 \overline{2} 0]$ direction on the polar free-standing (FS) GaN surfaces and on S1 sample with polar GaN QDs (symbol lines). Black solid and red dashed lines correspond to $+\mathrm{c}$ and $-\mathrm{c}$ GaN crystal polarity. XPD curve of S1 sample agrees well with $+\mathrm{c}$ orientation. (b) Experimental Ga 3d and Al 2p polar plots. The Al 2p polar plot agrees well with the Ga 3d plot and, therefore, $\mathrm{Al}$ atoms substitute the cation $(\mathrm{Ga})$ sites in $\mathrm{Al}_{0.5} \mathrm{Ga}_{0.5} \mathrm{~N}$ wurtzite lattice.

(0001) polarity of the polar GaN QDs is determined.

In Fig. 团 (b), the experimental polar plots of $\mathrm{Ga} 3 \mathrm{~d}$ and $\mathrm{Al} 2 \mathrm{p}$ core level intensities emitted in the m-plane are given. The photoelectrons emitted from $\mathrm{Al}$ atoms penetrate the thin GaN layer free of QDs. The Ga 3d curve of S1 sample is consistent with the Ga 3d curve of the polar FS-GaN(0001) sample. The close similarity of the shape of the two cation-induced polar plots indicates the same neighborhood of the $\mathrm{Ga}$ and $\mathrm{Al}$ atoms, which means that $\mathrm{Al}$ atoms occupy substitutional positions of $\mathrm{Ga}$ atoms in the $\mathrm{Al}_{0.5} \mathrm{Ga}_{0.5} \mathrm{~N}$ lattice. Therefore, the very good agreement between the XPD polar plots of S1 sample grown by MBE and FS-GaN(0001) sample grown by hydride vapour phase epitaxy (HVPE) [26] indicates a high structural quality of QDs and $\mathrm{GaN} / \mathrm{Al}_{0.5} \mathrm{Ga}_{0.5} \mathrm{~N}$ layers.

The polarity of semipolar free-standing GaN samples was investigated recently by XPD [27, 28]. The semipolar GaN crystals can be divided into two groups, depending on whether m-plane $(1 \overline{1} 00)$ or a-plane $(11 \overline{2} 0)$ is perpendicular to the semipolar surface. The $\{11 \overline{2} 2\}$ semipolar plane is obtained when tilting 
(a) N1s, [1100]

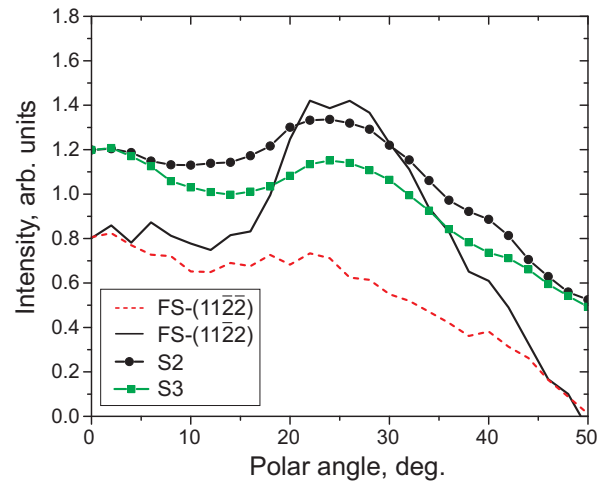

(b) N1s, [1̄1123]

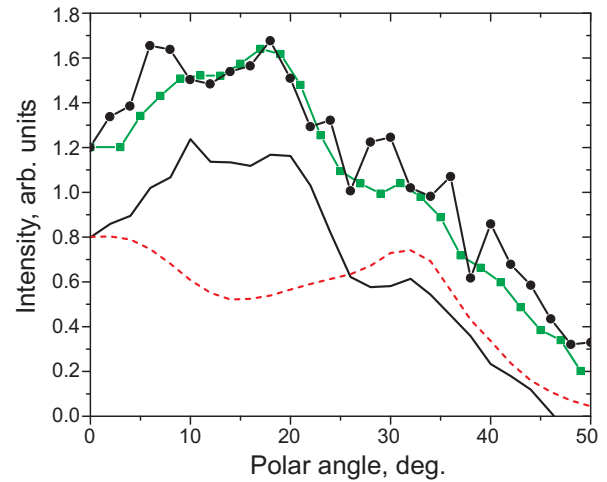

Figure 5: (Color online) Experimental polar plots of $\mathrm{N}$ 1s peak intensity measured along the (a) $<1 \overline{1} 00>$ direction and (b) $<\overline{1} \overline{1} 23>$ directions on free-standing GaN surfaces (solid and dashed lines) and on S2 and S3 samples (symbol lines). Normalized intensities were shifted by -0.2 and 0.2 down and up for FS and S2, S3 samples, respectively. It was confirmed that the buffer GaN epitaxial film on non-polar sapphire (sample S2) and GaN QDs (sample S3) have the $(11 \overline{2} 2)$ polarity.

the c-plane by $58.4^{\circ}$ or by tilting the a-plane by $31.6^{\circ}$ toward the c-plane. The m-plane is perpendicular to the $\{11 \overline{2} 2\}$ surface plane. The polar angle dependence of the photoelectron emission intensity is expected to be different for $\operatorname{GaN}(11 \overline{2} 2)$ and $(11 \overline{2} \overline{2})$ orientations in the polar angle range of $0^{\circ}-15^{\circ}$ along the [1̄123] direction or in the range of $19^{\circ}-26^{\circ}$ within the m-plane [28].

In order to clarify the polarity of the GaN QDs grown on semipolar GaN $\{11 \overline{2} 2\}$ layers, the reference sample S2 with only GaN epitaxial film and sample S3 with a multi GaN/ $\mathrm{Al}_{0.5} \mathrm{Ga}_{0.5} \mathrm{~N}$ QD layers were compared first [Fig. 1 (b), (c)]. The $\mathrm{N}$ 1s polar plots measured within the m-plane and along the [1̄123] direction are shown in Fig. 5 (a) and 5 (b), respectively. The normalized intensities were shifted by \pm 0.2 arbitrary units for clarity in the figures. Analyzing the dependencies, the following conclusions could be drawn: (i) photoelectron polar plot intensities from epilayers (S2) and QDs (S3) are similar and, therefore, the samples do have the same polarity; (ii) a good agreement of the polar plots

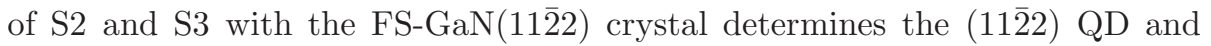


$\mathrm{GaN} / \mathrm{Al}_{0.5} \mathrm{Ga}_{0.5} \mathrm{~N}(11 \overline{2} 2)$ layers polarity.

In contrast to the polar samples, the polar plot intensities measured from semipolar QDs or epilayers are not perfectly correlated with the corresponding normalized intensities of the FS-GaN(11222) crystal. The MBE grown layers on (1100) sapphire seem to be less ordered than the HVPE grown GaN $\{11 \overline{2} 2\}$ crystals, most likely due to higher density of structural defects, including stacking faults and dislocations, being characteristic features of heteroepitaxial semipolar layers. Nevertheless, the peak position and the main features of the polar plots are sufficiently well correlated and the polarity of the (112̄2) QDs is clearly determined.

\section{Conclusions}

The polarity of wurtzite GaN QDs was determined non-destructively by X-ray photoelectron diffraction. GaN-based QDs heterostructures were grown on (0001) and (1100) sapphire substrates. Polar angle dependence of photoelectron emission intensities along specific directions in the GaN crystals were measured. The $+\mathrm{c}$ polarity of GaN QDs grown on sapphire (0001) substrate was determined. In case of semipolar GaN QDs, the $(11 \overline{2} 2)$ polarity was measured. The specifics of the polar plots of photoelectron intensities measured from $\mathrm{GaN}(0001)$ and $\mathrm{GaN}(11 \overline{2} 2)$ QDs were found consistent with those of the corresponding polar plots from free-standing GaN crystals. These findings offer attractive perspectives in the polar and semipolar GaN QD polarity analysis by a standard laboratory X-ray sources.

\section{Acknowledgments}

O. Romanyuk acknowledges the support by the Czech Science Foundation (GACR, project No. 15-01687S) and by the Ministry of Education, Youth and Sports of the Czech Republic (Grant LM2015088). J. Brault acknowledges the support by the French National Research Agency under the ANR Project (ANR- 
14-CE26-0025-01) "NANOGANUV" and T. Paskova acknowledges the support by NSF (DMR-1207075 and OISE-1458427).

\section{References}

\section{References}

[1] Y. Muramoto, M. Kimura, S. Nouda, Semicond. Sci. Technol. 29 (2014) 084004 .

[2] C. Pernot, M. Kim, S. Fukahori, T. Inazu, T. Fujita, Y. Nagasawa, A. Hirano, M. Ippommatsu, M. Iwaya, S. Kamiyama, I. Akasaki, H. Amano, Appl. Phys. Express 3 (2010) 061004.

[3] J. Grandusky, S. Gibb, M. Mendrick, C. Moe, M. Wraback, L. Schowalter, Appl. Phys. Express 4 (2011) 082101.

[4] Y.-H. Cho, B. J. Kwon, J. Barjon, J. Brault, B. Daudin, H. Mariette, L. S. Dang, Appl. Phys. Lett. 81 (2002) 4934.

[5] T. Huault, J. Brault, F. Natali, B. Damilano, D. Lefebvre, L. Nguyen, M. Leroux, J. Massies, Appl. Phys. Lett. 92 (2008) 051911.

[6] C. Himwas, R. Songmuang, L. S. Dang, J. Bleuse, L. Rapenne, E. Sarigiannidou, E. Monroy, Appl. Phys. Lett. 101 (2012) 241914.

[7] J. Brault, S. Matta, T. H. Ngo, M. Korytov, D. Rosales, B. Damilano, M. Leroux, P. Vennéguès, M. A. Khalfioui, A. Courville, O. Tottereau, J. Massies, B. Gil, Jpn. J. Appl. Phys. 55 (2016) 05FG06.

[8] J. Brault, B.Damilano, A.Kahouli, S.Chenot, M.Leroux, B. Vinter, J.Massies, J. Cryst. Growth 363 (2013) 282.

[9] J. Brault, D. Rosales, B. Damilano, M. Leroux, A. Courville, M. Korytov, S. Chenot, P. Vennéguès, B. Vinter, P. de Mierry, A. Kahouli, J. Massies, T. Bretagnon, B. Gil, Semicond. Sci. Technol. 29 (2014) 084001. 
[10] D. Rosales, T. Bretagnon, B. Gil, A. Kahouli, J. Brault, B. Damilano, J. Massies, M.V. Durnev, A.V. Kavokin, Phys. Rev. B 88 (2013) 125437.

[11] D. Huang, P. Visconti, K. M. Jones, M. A. Reshchikov, F. Yun, A. A. Baski, T. King, H. Morkoc, Appl. Phys. Lett. 78 (2001) 4145.

[12] Y. Xia, J. Brault, P. Vennéguès, M. Nemoz, M. Teisseire, M. Leroux, J.-M. Chauveau, J. Cryst. Growth 388 (2014) 35.

[13] H. Masui, S. Nakamura, S. P. DenBaars, U. K. Mishra, IEEE Trans. Elec. Dev. 57 (2010) 88 .

[14] A. Kahouli, N. Kriouche, J. Brault, B. Damilano, P. Vennéguès, P. de Mierry, M. Leroux, A. Courville, O. Tottereau, J. Massies, J. Appl. Phys. 110 (2011) 084318.

[15] J.Brault, S. Matta, T.-H. Ngo, D. Rosales, M. Leroux, B. Damilano, M. A. Khalfioui, F. Tendille, S. Chenot, P. D. Mierry, J. Massies, B. Gil, Mat. Sci. in Semicond. Proces. in press (2016) http://www.sciencedirect.com/science/article/pii/S136980011630035X.

[16] M. Seelmann-Eggebert, J. Weyher, H. Obloh, H. Zimmermann, A. Rar, S. Porowski, Appl. Phys. Lett. 71 (1997) 2635.

[17] O. Romanyuk, S. Fernández-Garrido, P. Jiříček, I. Bartoš, L. Geelhaar, O. Brandt, T. Paskova, Appl. Phys. Lett. 106 (2015) 021602.

[18] S. Fernandez-Garrido, X. Kong, T. Gotschke, R. Calarco, L. Geelhaar, A. Trampert, O. Brandt, Nano Lett. 12 (2012) 6119.

[19] K. Hestroffer, C. Leclere, C. Bougerol, H. Renevier, B. Daudin, Phys. Rev. B 84 (2011) 245302.

[20] D. Cherns, L. Meshi, I. Griffiths, S. Khongphetsak, S. V. Novikov, N. Farley, R. P. Campion, C. T. Foxon, Appl. Phys. Lett. 92 (2008) 121902. 
[21] X. Kong, J. Ristic, M. A. Sanchez-Garcia, E. Calleja, , A. Trampert, Nanotechnology 22 (2011) 415701.

[22] M. D. Brubaker, I. Levin, A. V. Davidov, D. M. Rourke, N. A. Sanford, V. M. Bright, K. A. Bertness, J. Appl. Phys. 110 (2011) 053506.

[23] J. Wei, R. Neumann, X. Wang, S. Li, S. Fündling, S. Merzsch, M. A. M. Al-Suleiman, U. Sökmen, H.-H. Wehmann, A. Waag, Phys. Status Solidi C 8 (2011) 2157.

[24] M. den Hertog, F. González-Posada, R. Songmuang, J. Rouviere, T. Fournier, B. Fernandez, E. Monroy, Nano Lett. 12 (2012) 5691.

[25] J.-L. Rouviere, C. Bougerol, B. Amstatt, E. Bellet-Almaric, B. Daudin, Appl. Phys. Lett. 92 (2008) 201904.

[26] O. Romanyuk, P. Jiříček, T. Paskova, I. Bieloshapka, I. Bartoš, Appl. Phys. Lett. 103 (2013) 091601.

[27] O. Romanyuk, P. Jiříček, T. Paskova, I. Bartoš, J. Appl. Phys. 116 (2014) 104909 .

[28] O. Romanyuk, P. Jiříček, T. Paskova, I. Bartoš, J. Mater. Res. 30 (2015) 2881.

[29] M. Korytov, M. Benaissa, J. Brault, T. Huault, T. Neisius, P. Vennéguès, Appl. Phys. Lett. 94 (2009) 143105.

[30] The $[11 \overline{2} 0]$ direction or $(1 \overline{1} 00)$ plane lies between the hexagonally-aligned the $(1,0)$ and $(0,1)$ LEED spot maxima, whereas the [1100] direction or (112̄0) plane crosses the LEED spot maxima. There is a misprint in Ref. 25, Fig. 1(a)

[31] S. Ploch, T. Wernicke, J. Thalmair, M. Lohr, M. Pristovsek, J. Zweck, M. Weyers, M. Kneissl, J. Cryst. Growth 356 (2012) 70. 
[32] M. Sawicka, C. Chèze, H. Turski, J. Smalc-Koziorowska, M. Kryśko, S. Kret, T. Remmele, M. Albrecht, G. Cywiński, I. Gregory, C. Skierbiszewski, J. Cryst. Growth 377 (2013) 184.

[33] O. Romanyuk, I. Bartoš, Surf. Sci. 603 (2009) 2789. 